\title{
Integrating the Circuit
}

Little more than a decade after John Bardeen, Walter Brattain, and William Shockley first demonstrated the transistor at Bell Laboratories in 1948, another revolution occurred in electronics. It became possible to form entire electronic circuits on a single thin slice of silicon and use them to control and amplify an electric current. Modernday integrated circuits are composed of numerous interconnected electronic components-transistors, diodes, capacitors, or resistors-patterned onto the surface of a tiny chip about $1 / 20$ th of an inch square.

During World War II, engineers realized the need to reduce the size of electronic equipment to accommodate advances in radar, recording media, piezoelectric crystals, and other technologies. Several research teams studied ways to miniaturize circuit construction.

At this time, electronic circuits were composed of separate devices, each encapsulated in ceramic, metal, plastic, or glass and joined by welding or soldering. Printed circuit paths were etched in a metal foil bonded to a ceramic block; these paths electrically linked separate components mounted on the block.

With the invention of the transistor, solid-state devices began the rapid and near-total replacement of vacuum tubes in electronic equipment. Transistors were much less expensive, much smaller, used less power, did not burn out in service at nearly as high a rate, and were generally more reliable than vacuum tubes. Small transistors attached to printed circuit boards made a much more compact package than vacuum tubes would ever allow. As early as 1952-long before transistors gained widespread acceptance in the electronics industry-Geoffrey W.A. Drummer of Britain's Royal Signals and Radar Establishment proposed the possibility of creating "electronic equipment in a solid block with no connecting wires."

While such circuits could be laid down on any electrically inert substrate such as ceramic or glass, the success of transistors also suggested the enormous potential of semiconductor materials for other uses. A key property of semiconductors is that manufacturers can impregnate tiny amounts of impurities into precisely selected areas, creating doped zones with excess positive or negative charge carriers.

In the late 1950s, improvements in silicon purification allowed producing material with high-enough quality for semiconductor devices. Germanium was the first material used for semiconductors because methods of purifying it sufficiently had been known as early as World War II. However, silicon was much more abundant and less expensive than germanium. And, because silicon has a larger band gap than germanium, it produces less noise at a given temperature, and it retains its semiconducting properties at higher temperatures. By the end of the 1950s, silicon showed itself to be the best semiconducting material for mass production.

Many transistors can be fabricated simultaneously on a single thin wafer of high-quality silicon, and the wafers can then be cut apart into individual chips. In 1958, Jack Kilby of Texas Instruments realized the practicality of combining passive electronic devices (resistors and capacitors) and active devices (transistors and diodes) all on a single semiconducting substrate. That year, the first "monolithic integrated circuit," which contained at least one element within the substrate itself, was successfully demonstrated.

Working independently of Kilby, Robert Noyce, Jean Hoerni, and Gordon Moore at Fairchild Semiconductor Company used planar technology and diffused junctions to develop a silicon integrated circuit. They used multiple diffusion steps to place the desired impurities in the surface of a silicon crystal, laying down the circuit paths. Their technique is the basis for most silicon integrated circuit processing today. Kilby is usually credited with developing the concept for integrated circuits, while Noyce and co-workers are credited with the fabrication method.

After the groundwork for integrated circuits was laid, a number of companies and government research establishments began to push for further development. At that time, computers used hundreds of thousands of transistors, so integrated circuits (then called "micrologic elements") seemed the best way to make computers smaller but more powerful.

In the early 1960 s, U.S. government integrated circuit research focused on developing a compact, lightweight electronic guidance system for the Minuteman II intercontinental ballistic missile. The circuits had to be accurate, reliable, and lightweight. Because the Minuteman missiles were to be stored for long periods in deep underground silos, the circuits also had to resist moisture, temperature fluctuations, and the effects of aging. Each Minuteman II guidance computer eventually required nearly 3,000 integrated circuits.

Integrated circuits are electrically faster than conventional circuits. Early integrated circuits operated at switching speeds up to one million bits per second; by 1989 they operated in excess of ten million bits per second, and they continue to increase by orders of magnitude. As switching speed increases, reducing the lengths of connecting pathways becomes more crucial to increase speed.

One important question regarding the first integrated circuits was whether their reliability decreased as their complexity increased. The failure of complex, nonrepairable circuits sooner than simple devices would have severely constrained progress in electronics, but it was discovered that an integrated circuit's reliability is independent of its complexity.

The first mass-produced integrated circuit consisted of about 10 individual components on a $3 \mathrm{~mm} \times 3 \mathrm{~mm}$ silicon chip. The development of large-scale integration (LSI) in the early 1970 s made it possible to pack thousands of components on a chip of roughly the same size and for the same cost. Very large-scale integration (VLSI), developed in the 1980s, vastly increased the circuit density, yielding more than three million transistors on a chip less than $2 \mathrm{~cm} \times 2 \mathrm{~cm}$. Now research is moving into ultra-large-scale integration (ULSI).

In 1971 an Intel Corporation team introduced the first microprocessor, an integrated circuit containing all the control circuitry, logic, and arithmetic necessary to carry out every function of an entire digital computer's central processing unit-on a single $5 \mathrm{~mm} \times 5 \mathrm{~mm}$ silicon chip. This opened the door for massive expansion in the small electronics industry.

The demand for microprocessors was spurred on by successful applications in manufacturing, such as one-armed robots for spot welding and numerically controlled lathes. This large demand encouraged high-volume production of integrated circuits and microprocessors, leading to more efficient processes and dramatic drops in cost. Lower prices in turn led to use of microprocessors in household appliances and automobiles, items for which electronic controls had previously been too expensive.

Today's rapid development of optical devices, maturation of compound semiconductors, and continued refinement of device processing is unfolding yet another generation of smaller, faster, more versatile circuitry.

Kevin J. Anderson 


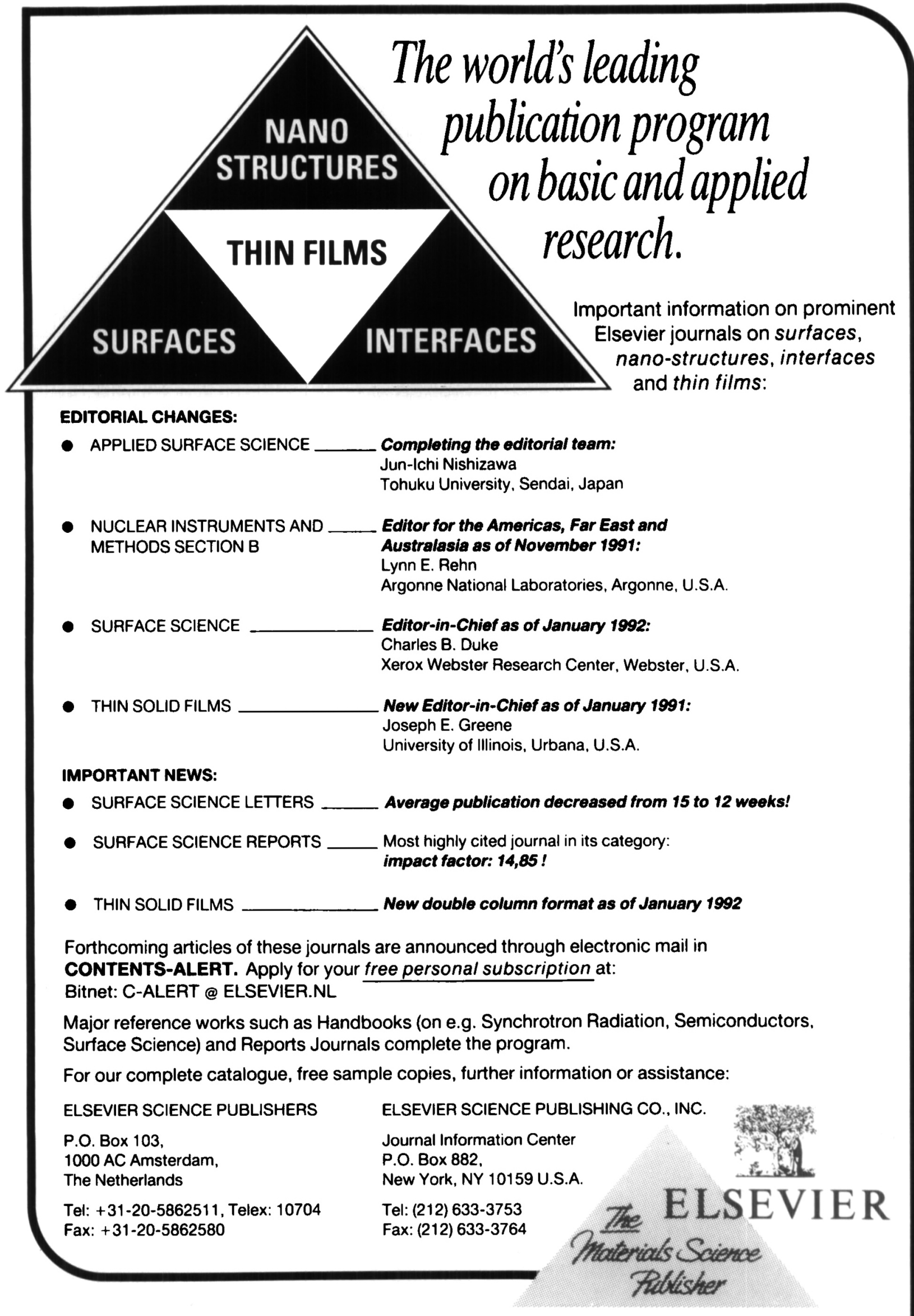

